\title{
Cuidado de sí mismo en el bachillerato, microdispositivos pedagógicos de transformación emergente
}

Care of the self in the upper secondary education,
pedagogical microdevices for emergent transformation

\section{Mónica CRistina Ramírez Montes De OcA*}

El bachillerato en México enfrenta una emergencia formativa demandada por sus estudiantes, en la cual diversas dimensiones que los configuran como sujetos pueden ser reconocidas. Responder a ello es posible a partir de la configuración de microdispositivos pedagógicos en los que se concretan prácticas para el cuidado de sí mismo; la apertura, sedimentación y entrecruce que caracteriza a estos dispositivos produce unidades que permiten el acceso a la verdad sobre sí mismo, la construcción de sentido y la transformación de los sujetos. Con base en un trabajo genealógico y de enfoque interpretativo, este artículo analiza diversas prácticas realizadas en una escuela preparatoria del Instituto de Educación Media Superior cuya finalidad es identificar condiciones, entre lo tradicional e inédito, que hacen posible la configuración de estos microdispositivos. Los resultados revelan que en algunos de estos existe una estructura y duración, pero en otros hay una configuración efímera. No obstante, lo importante son las respuestas de los profesores, las conexiones entre lo intempestivo y lo sedimentado escolar, y cómo los estudiantes, en distinta medida, logran un gobierno sobre sí expresado en acciones para transformar su existir. La escuela, así, puede significar un acontecimiento importante en sus vidas.

In Mexico, upper secondary education (high-school) faces several challenges, among them a "formative emergency" as students, understood as the multiplicity of dimensions that configure them as subjects in order to get recognition. One of the possible answers to this emergency could be the configuration of pedagogical micro-devices oriented to develop practices of self-care as the opening, sedimentation and cross-linking that characterizes that kind of devices could produce meeting points that allow access to the Truth about oneself, the construction of meaning and the transformation of the subjects.Product of genealogical work and an interpretative approach, is the analysis of various practices carried out in a High School which belongs to the Institute of Higher Secondary Education, to locate conditions, between the traditional and unpublished, which make possible the configuration of such micro-devices. As a result, structure and duration are found in some of them, in others, an ephemeral configuration. Along with all these processes, the most important are Teachers' answers, as well as the connections between the unexpected and the leaks from school sedimentation, as well as the folds that all this allows in the students, who eventually achieve a government over themselves expressed in actions to transform their existence. If all of that is achieved, schools may be an important event in young people lives.

\author{
Palabras clave: \\ cuidado de sí \\ mismo, \\ bachillerato, \\ microdispositivos \\ pedagógicos, \\ escuela
}

\section{Keywords:}

care of the self,
upper secondary
education,
pedagogical
microdevices,
school

Recibido: 28 de febrero de 2018.| Aceptado para su publicación: 3 de septiembre de 2018. DOI: 10.31391/S2007-7033(2018)0051-009

\footnotetext{
* Doctora en Pedagogía por la Facultad de Filosofía y Letras de la UNAM. Profesora en el Centro de Estudios Superiores de la Ciudad de México. Trabaja como consultora curricular independiente. Sus líneas de investigación abordan la pedagogía, la filosofía y la didáctica. Correo electrónico: monixrmdo@gmail.com
} 


\section{INTRODUCCIÓN}

1 bachillerato en México se ha caracterizado en las últimas décadas por tener

grandes dificultades para superar problemas de rezago, reprobación, falta de cobertura, deserción e impacto adecuado en el aprovechamiento de las poblaciones juveniles que conforman sus filas. Esto, aunado a un contexto de grandes cambios a nivel cultural, tecnológico, de desigualdad social y desconfianza creciente en las instituciones, coloca a la escuela frente a múltiples retos por cumplir si, efectivamente, pretende dar respuestas contemporáneas a los actores que en ella convergen.

A pesar de esas dificultades, acudir a escuelas de este nivel aún es valorado por los jóvenes, pues depositan expectativas materiales, laborales y de continuidad educativa (Tapia, 2012), de sociabilidad juvenil (Guerrero, 2000) o la ampliación de sus horizontes ante la posibilidad de otros procesos formativos y de subjetivación (Weiss et al., 2009, 2012; Ramírez, 2012).

Este trabajo retoma el último punto de una investigación anterior (Ramírez, 2012) que revela que jóvenes que acuden a bachilleratos con altos niveles de regulación disciplinaria demuestran distintas formas de hartazgo, rechazo y malestar, pues diversas dimensiones de su configuración subjetiva son negadas. Sin embargo, en los intersticios escolares, esos jóvenes son capaces de asimilar prácticas que les permiten expresar sus sentires, necesidades, inquietudes, saberes, formas de ser y de relacionarse. Esto hace evidente una emergencia formativa de amplias dimensiones que debe ser atendida mediante el reconocimiento de sus expresiones culturales, artísticas, deportivas, de consumo, o bien, en otras maneras de socialización, ejercicio de la libertad y creación de sí mismo.

Desde este planteamiento, surgen las preguntas ¿qué prácticas irrumpen en la escuela? ¿Sus capturas intersticiales indican propuestas distintas de los profesores? ¿Estas atienden la emergencia formativa demandada por los jóvenes de la escuela del nivel medio? ¿Es posible la producción y actualización de prácticas de cuidado de sí mismo en el espacio escolar mediante dispositivos pedagógicos para una formación distinta de los sujetos?

Tomando estas interrogantes como base, el supuesto de trabajo señala que, mediante la configuración de dispositivos pedagógicos que posibiliten el cuidado de sí mismo, desde una perspectiva foucaultiana-deleuziana, es factible atender dicha emergencia, pues su carácter estratégico, articulador y heterogéneo permite conectar y producir otras prácticas, saberes, formas de relación y también subjetividades, sobre todo cuando los sujetos en el ejercicio de su libertad logran plegarse para conocerse y configurar otros modos de ser (Deleuze, 1987; Foucault, 2002).

Estos dispositivos se producen en la escuela preparatoria Otilio Montaño, perteneciente al Instituto de Educación Media Superior de la Ciudad de México (IEMS), cuya procedencia se define por una lucha sociopolítica y por oponerse a planteamientos de orden hegemónico-tradicional mediante su modelo educativo. El objetivo de nuestra investigación es analizar diversas prácticas realizadas en esa escuela e identificar si en el cruce de lo tradicional e inédito configuran dispositivos pedagógicos que permiten el cuidado de sí mismo en los jóvenes estudiantes. 


\section{MARCO TEÓRICO-METODOLÓGICO}

\section{La genealogía y los dispositivos}

La tarea genealógica articula conceptos como origen, procedencia y emergencia para mantener una actitud crítica sobre los acontecimientos, cuyo devenir discontinuo y contingente se forma de luchas diversas, ámbitos y tramas yuxtapuestas en diferentes niveles. Se trata principalmente de comprender las condiciones históricas de su posibilidad (Foucault, 1990, 1992, 1999). Para tal finalidad se utilizan los dispositivos, los cuales dan cuenta del entrecruzamiento múltiple de componentes, procesos, plegamientos, líneas en reposo o transformación que dan forma a cada realidad, así como del agenciamiento o unidad mínima desde la cual las multiplicidades funcionan (Deleuze, 1999; Deleuze y Guattari, 2002).

Las líneas que componen los dispositivos son:

- Líneas de visibilidad y enunciación, o del saber. Dependiendo de cada estrato o formación histórica, se reparte aquello que puede ser dicho o visto, como agenciamientos prácticos de tipo ético, político, estético, científico, etcétera, mediante los que se produce verdad (Deleuze, 1987).

- Líneas de fuerza o de poder. Son múltiples y atraviesan puntos, instituciones, discursos, sujetos; sus efectos son producto de "disposiciones, maniobras, tácticas, técnicas, funcionamientos” (Deleuze, 1987, p. 51) y poseen dos dimensiones: el diagrama, presente en todo el campo social, "es la presentación de fuerzas propias de una formación; la distribución de poderes de afectar y ser afectado" (Deleuze, 1987, p. 70), y los microdispositivos, de carácter múltiple, heterogéneo o difuso; estos pueden efectuar el diagrama y, a la vez, el diagrama puede difundir los efectos que ellos producen en otros microdispositivos del mismo campo social. En este trabajo retomamos solo la segunda dimensión.

-Líneas de fractura o subjetivación. Son producto del trabajo del sujeto consigo mismo, quien, al poseer poder, produce un pliegue, expresión de una fuerza capaz de afectarse y regularse a sí misma desde el interior (Deleuze, 1987). El pliegue es variable, pues mantiene relación con cuatro aspectos: con una conducta moral; con reglas divinas, naturales, civiles, estéticas o racionales; con técnicas trabajadas sobre sí; y con aquello que el sujeto espera: felicidad, eternidad, perfección, libertad, salud, renuncia o muerte (Foucault, 2013).

Las tres líneas poseen dos movimientos que no se separan: de sedimentación y de fuga. Los primeros "crean estructuras estables, compactas, funcionales" [y los segundos] son fuerzas y funciones desestratificadas [...] son una especie de mutación, de creación" (Deleuze y Guattari, 2002, pp. 153, 512, 517). Por ello, los dispositivos no son infranqueables; denotan gran complejidad en su composición, sistematización y desciframiento; no obstante, producen verdades, crean y transforman aspectos vitales de la realidad; modifican y pueden ser modificados para crear algo diferente. 


\section{Cuidado de sí mismo y su implicación pedagógica}

En su interés por las prácticas de subjetivación, Foucault estudia la relación entre juegos de verdad o reglas desde las cuales lo que los sujetos dicen se inscribe en el campo de lo verdadero o falso (Foucault, 1999), y las tecnologías del yo, como acciones realizadas por los sujetos sobre su cuerpo, alma, pensamientos, conducta o formas de ser para obtener una transformación de sí mismos a fin de ser felices, puros, sabios o inmortales (Foucault, 1990). Esa articulación genera una pauta de relación con uno mismo que se materializa según las condiciones de cada época.

Una manifestación importante de la relación anterior Foucault la encuentra en los siglos I y II de la época grecorromana, cuando estas tecnologías vividas bajo el principio de epimeleia heautou o cuidado de sí mismo se expresan de manera diversa y cumplen sobre todo un papel ethopoyético (Foucault, 2010) o de formación ética.

En un primer momento, cuidar de sí mismo implica cuidar del alma; no como sustancia, sino como sujeto de acción, de relaciones y actitudes; tomar una posición no instrumental e irreductible frente a la realidad, los otros, el lenguaje, los acontecimientos y frente a sí. Para ello, es importante cumplir con el gnothi sauton o conocerse a sí mismo y acercarse a una verdad trascendente (Foucault, 2002). En otro momento, significa cuidar de la propia vida, del estilo dado a la existencia, lo que demanda, además de un conocimiento de sí mismo, el poder dar cuenta de sí, como la relación entre el logos (la razón) y la manera de vivir, hacer y pensar (Foucault, 2010).

Con estos rasgos, cuidar de sí mismo se vuelve un imperativo general disperso como dispositivo cuyo fin es "el perfecto gobierno de uno mismo: una especie de relación política entre uno y uno mismo" (Foucault, 2013, p. 148); "exige coraje y sobre todo un cuidado del mundo y de los otros" (Gros, citado en Foucault, 2010, p. 357); por tanto, es un cuidado que, si bien hace referencia al compromiso con respecto a sí mismo, encierra un lazo y un compromiso con los otros.

En el diagrama de esa época, el cuidado de sí puede expresarse de formas diversas; una de ellas se da en la relación pedagógica en la cual la presencia del maestro es fundamental para que se lleve a cabo la reforma del individuo (Foucault, 2002). Para ello, es importante que él mismo efectúe su cuidado e impulse con su ejemplo a que sus alumnos lo concreten en el tránsito de dos momentos:

-El primero es la mathesis. Al conocer sobre cosas, dioses u hombres, los alumnos deben ser un término permanente para formular así preceptos de conducta que resulten en un ethos, en otras maneras de ser y comportarse.

-El segundo es la askesis. La comunicación de los discursos de verdad ha de seguir reglas éticas en la escritura, la lectura o habla. De este modo se significa, valora y da forma a la experiencia personal. Se ponen en acción aquellos discursos por medio de pruebas o ejercicios que permitan cuidar aspectos corporales, formas de pensamiento, pasiones, deseos o afectos. Son prácticas que permiten enfrentar vicisitudes, fortalecer el gobierno de sí, incrementar la comprensión y posibilidades de estar en el mundo y su recreación.

Por lo anterior, el trabajo pedagógico, además de transmitir saberes, habilidades o capacidades, se centra en la formación humana. Esta se expresa en el devenir constante y abierto de los sujetos pedagógicos (profesores y estudiantes), en las múltiples 
posibilidades para ser, actuar, pensar, transformarse y construir sentido; se orienta siempre por las metas, prescripciones, dilemas y contingencias de la experiencia de los mismos sujetos.

Para que estos procesos, prácticas y transformaciones sean posibles, es necesario que se inscriban en cierto dispositivo pedagógico. En el campo educativo, el uso dado a los dispositivos pedagógicos es diverso (Beillerot, citado por Souto, 1999; Elizondo, 2004; Castañeda, 2004; Yurén, 2008; Estrada y Araujo, 2008; Valencia, 2008; Álvarez, 2012). En nuestro caso, los utilizamos como herramienta teórico-metodológica para acceder a la construcción, análisis y comprensión compleja y multidimensional de prácticas y procesos que hacen posible la formación de los sujetos pedagógicos, guiada por el principio del cuidado de sí mismo.

No pretendemos la réplica de las prácticas de los siglos I y II, sino su actualización en un dispositivo escolar de nivel medio superior, pues "la relación con uno mismo no cesa de traducirse, metamorfoseándose [...] no cesa de renacer en otro sitio y en otra forma" (Deleuze, 1987, p. 136). En este entendido, el dispositivo pedagógico lo comprendemos como la articulación histórica, múltiple y heterogénea de discursos educativos, reglamentaciones, principios filosóficos, éticos, morales; de enunciados científicos y disciplinarios; de formas de gestión e instituciones. Tal articulación es definida por el ejercicio del poder en la regulación de las prácticas y los procesos de los sujetos pedagógicos.

Desde el plano del diagrama, el dispositivo pedagógico responde a una urgencia social, económica, política, religiosa, cultural, etcétera, al crear instituciones y dar forma al campo educativo. Desde la dimensión micro-, las fuerzas y relaciones del diagrama se recrean en contextos particulares como la escuela; en ella se ubica el actuar cotidiano y la constitución de los sujetos pedagógicos; se incorpora sedimentaciones o se producen agenciamientos, entre los que está el cuidado de sí mismo.

\section{La escuela como dispositivo pedagógico}

Por la amplitud del análisis genealógico del nivel medio como dispositivo escolar en su dimensión diagramática, solo exponemos ciertos rasgos:

La procedencia de este dispositivo se configura por varios acontecimientos y dispersión de fuerzas. En el presente esto se refleja al ver desdibujados los fundamentos, estrategias y saberes en que se apoyaba. Asimismo, al ser trastocada por realidades intempestivas, muchas veces indecibles, desconcertantes o conflictivas para sus habitantes, estos se tienen que mover entre el plano escolar y no escolar del dispositivo, es decir, entre percepciones homogeneizantes y estereotipadas de las situaciones educativas que le permiten seguir siendo un aparato burocrático-estatal y entre lo que la desafía como proyecto clásico: formas y flujos de vida múltiples, modos de gestión, intercambios comunicativos, ritmos de trabajo, de pensamiento y creación (Duschatzky, 2007; Duschatzky y Sztulwark, 2011).

Lo anterior demanda la gestión de nuevas prácticas para pensar, nombrar y trabajar con lo diferente, con aquello que busca hacer nuevas conexiones o, como hemos dicho, configurando microdispositivos pedagógicos inéditos, que propicien otras posibilidades pedagógicas. 


\section{Enfoque, herramientas y momentos analíticos de la investigación}

El enfoque que sigue nuestra investigación es interpretativo, pues permite la descripción y comprensión analítica por niveles de una realidad múltiple, no para descubrir esencias o comienzos, como lo señala Foucault (1967/1969), sino para construir la materialidad histórica de los objetos y los sujetos. Ello se produce en una articulación entre interpretaciones ya realizadas, donde relaciones de violencia como de elucidación son posibles, pues al presentarse diversas fuerzas constructoras de la verdad, otras formas de intersección narrativa son configuradas y con ello nuevos sentidos.

Interpretar entonces es un "acontecimiento que funda un nuevo Logos, un nuevo espacio de inteligibilidad desde el cual todo el 'mapa' de la cultura se recompone y lo hace por la imaginación y por la construcción de un 'relato'” (Grüner $1967 / 1969)$. En este caso, producto de diversos textos institucionales, contextuales y normativos, así como de aquellos ensayos interpretativos que estudiantes y profesores producen para constituirse.

Para esta tarea, nos apoyamos en la indagación documental de diferentes textos a fin de comprender la emergencia histórica del dispositivo escolar, además de las condiciones de posibilidad del IEMS y sus escuelas. En cuanto a la estrategia etnográfica, planteamos las siguientes acciones:

Observación. Construimos una guía que nos permite orientar la mirada en el territorio escolar y hacer registros desde las tres líneas que conforman los dispositivos. Como producto, obtenemos registros de prácticas inesperadas o programadas, de encuentros con algunos actores, croquis del edificio escolar, fotografías intra- y extraescolares, así como algunos videos.

Cuestionario. Diseñamos y aplicamos un cuestionario a 52 estudiantes de quinto y sexto semestre, 30 de la modalidad escolar y 22 de la modalidad semiescolar. El criterio de aplicación son los referentes que poseen, producto del tiempo y las experiencias construidas en su trayecto escolar. El cuestionario tiene 15 preguntas, ocho de tipo abierto, que indagan sobre los microdispositivos más valiosos y significativos propuestos por sus profesores; buscamos el vínculo entre prácticas y efectos provocados en su subjetividad. Las otras siete son de tipo cerrado y abordan rasgos de su condición familiar y escolar.

Entrevistas. Constituyen un espacio de interpelación donde los sujetos pueden hablar de manera reflexiva sobre la configuración de su subjetividad, articulada a los procesos y prácticas pedagógicas vividas dentro de la escuela, no como un "acto de biografía confesional o de relatos de los acontecimientos históricos que se produjeron en la vida [...] sino para mostrar que hay una relación entre el discurso racional, el logos que somos capaces de utilizar y nuestra manera de vivir" (Foucault, 2001, p. 97); por ello, permiten ubicar prácticas de cuidado de sí como aquellas de corte más sedimentado. Las entrevistas se hicieron a tres actores escolares:

-Entrevistas a 34 estudiantes de ambas modalidades: 27 de ellos, al contestar el cuestionario, expresaron mayores elementos de transformación subjetiva producto de los microdispositivos propuestos; las otras siete entrevistas son producto del hallazgo de actividades localizadas durante la observación, por lo que los estudiantes salen del encuadre inicial: cinco son 
de cuarto semestre, uno de segundo y uno es independiente, pues rebasa los tiempos regulares de conclusión del bachillerato.

- Las entrevistas contenían 17 preguntas de tipo abierto, las cuales pretendían que los sujetos hicieran contacto con aquellas dimensiones de su subjetividad transformadas o configuradas desde las propuestas hechas; qué reglas y verdades las soportan, en qué consiste la propuesta, el tipo de relaciones con los profesores y estudiantes, y el impacto que tiene en su estar en la escuela.

-Entrevistas a 11 profesores de la modalidad escolarizada y seis de la semiescolarizada. Elegimos a los más nominados en los cuestionarios, excepto dos, quienes se hallaban en el trabajo de observación. La entrevista estuvo compuesta de 13 preguntas abiertas que indagan sobre el formato, intenciones e impacto que, a su parecer, aportan sus propuestas a la subjetividad de sus estudiantes; otras tres preguntan por su formación, experiencia profesional y con configuraciones formativas propuestas para conseguir la autonomía de los estudiantes.

-Tres entrevistas a personal administrativo. La intención fue ampliar los elementos de análisis respecto del contexto, de las modalidades educativas y la dinámica del plantel. El número de preguntas varía, pues se formulan en el momento oportuno.

Para hacer referencia a los datos de las entrevistas, guardamos el anonimato de los sujetos utilizando una clave formada por una letra y un número aleatorio. Para profesores, usamos la letra P y P* cuando es más de un profesor, la J para los estudiantes y la A para los administrativos. Para referir datos del cuestionario y de los registros de observación, utilizamos $\mathrm{C}$ y RO, respectivamente.

La indagación pasa por dos momentos analíticos que nos permiten darle orden:

Sistematización y primer análisis. De los registros de observación, cuestionarios y entrevistas, obtuvimos distintos ordenamientos: croquis del edificio escolar y descripciones de prácticas halladas; del contenido base de las respuestas abiertas del cuestionario, construimos series por ámbitos abordados y de las cerradas, frecuencias por elección.

Para las entrevistas, tomamos las categorías de mathesis y askesis para analizar qué principios, posturas y prácticas guían el ajuste de los conocimientos efectuados por los profesores, su relación pedagógica, sus intencionalidades formativas, modo de autoridad ejercida, entre otros aspectos.

Por cada estudiante entrevistado, generamos una matriz analítica, que retoma los cuatro aspectos del plegamiento subjetivo, e identifica relaciones entre prácticas, códigos, verdades y transformaciones que visibilicen prácticas para el cuidado de sí mismo; el papel que en ello juegan los profesores y diversas disposiciones institucionales, personales o relacionales.

Construcción de categorías. Es el resultado del entrecruzamiento de las categorías teóricas, el ordenamiento empírico y el trabajo de descripción e interpretación analítica. Por medio de ellas, mostramos las capturas hechas por profesores y estudiantes de las líneas que les permiten, en mayor o menor grado, generar prácticas para el cuidado de sí mismo y, con ello, procesos de transformación. 


\section{RESULTADOS}

\section{La línea se extiende a Topilejo}

La creación de una nueva preparatoria en la delegación Iztapalapa (en 1999) fisura el dispositivo escolar dominante en la Ciudad de México. Esto, debido a múltiples procesos y acontecimientos de corte demográfico, político, social y educativo en los que sujetos de esos ámbitos luchan por la transformación de su entorno, la elevación de su dignidad humana y ciudadana (impidiendo la reactivación de una cárcel), así como por la emancipación social de sus jóvenes.

Tal fisura alcanza a las demás delegaciones con la intención de solventar la gran demanda del nivel medio, principalmente entre los sectores más excluidos de la ciudad. Así, la línea se extiende a los pueblos y barrios ancestrales de la delegación Tlalpan, y es San Miguel Topilejo el pueblo ganador en la contienda por albergar una preparatoria (en 2001) que dará educación a jóvenes de esos pueblos, incluyendo algunos de Xochimilco (A1).

La preparatoria Otilio Montaño visibiliza en su arquitectura, equipamiento profesional, material y de recursos pedagógicos una línea de saber acorde con lo plasmado en su modelo educativo, el cual busca solventar "necesidades y aspiraciones educativas de los estudiantes" (IEMSDF, 2006); no obstante, depende del gobierno citadino en turno para contar con los recursos suficientes.

Como en todas las escuelas del IEMS, Otilio Montaño tiene dos modalidades educativas, la escolarizada y la semiescolarizada. A la primera acuden de lunes a viernes estudiantes de entre quince y veintiún años de edad para recibir clases, asesorías, tutorías y participar en las actividades que de estas se desprendan, en turno matutino o vespertino. Durante el ciclo 2015-2016 "A" son atendidos 1,001 estudiantes por 63 docentes-tutores-investigadores, titulados y con tiempo completo. En la segunda acuden 390 estudiantes de entre diecisiete y cincuenta años o más, quienes combinan trabajo presencial mediante asesorías y trabajo autónomo; por ello, asisten dos o tres veces a la semana para ser atendidos por 18 asesores contratados por horas, cuya tarea central es orientar el trabajo autónomo de los estudiantes.

Algunos rasgos de los estudiantes de esta preparatoria se relacionan con su heterogeneidad, ya que, al ingresar por sorteo, no hay filtros etarios, sociales o académicos; ello rompe con procesos de normalización (De Alba, 2011) y abre espacios y múltiples posibilidades a sujetos con diferentes niveles socioeconómicos, capacidades, experiencias y visiones del mundo. En general, los estudiantes de ambas modalidades tienen una condición familiar y económica que les permite contar con redes de apoyo humano y recursos materiales suficientes para enfrentar sus compromisos escolares.

Los once profesores más nominados en la modalidad escolarizada tienen una larga trayectoria profesional dentro del nivel medio (excepto uno); siete participan desde el comienzo del proyecto en Topilejo, lo que habla del cúmulo de sus experiencias y herramientas de distinto orden, así como de su posicionamiento frente al modelo educativo. Por su parte, los profesores de la modalidad semiescolarizada tienen en promedio seis años dando clase en el nivel medio y de tres a seis años en el IEMS; por las características de la modalidad, lo propuesto por ellos se centra sobre todo en el trabajo de asesoría y acompañamiento para la certificación. 
Los resultados que siguen se enfocan en dos microdispositivos cuya diferencia se presenta en el nivel de estructura, temporalidad y riqueza formativa, ya que, por cuestiones de espacio, no abordamos aquello que responde a lógicas de lo estático, instrumental o de normalización, en las que prevalece la indiferencia, el descuido o la inmovilidad; estas situaciones también están presentes en la configuración del dispositivo escolar y dificultan, por supuesto, la producción de prácticas para el cuidado de sí mismo.

Filos-café, el espacio de vidas compartidas

Un espacio montado para funcionar como cafetería escolar no es tal, pues entre las múltiples actividades que en él se realizan está el encuentro semanal de tutoría de un grupo aproximado de quince estudiantes con un profesor de filosofía y una profesora de literatura al que denominan Filos-café. Diferencias en el vestir, hablar y conducirse son algunas marcas de distinción cultural, social e identitarias de este grupo de estudiantes, quienes, sentados junto con los profesores alrededor de dos mesas contiguas, beben café, comen panecillos y abordan uno de los temas anotados en una pizarra: autoconocimiento, empatía, comunicación asertiva, relaciones interpersonales, toma de decisiones, manejo de tensiones, pensamiento crítico y creativo, respeto de sí mismo y del otro (RO). Sin detallar la dinámica del grupo, analizamos el microdispositivo desde sus actores: "El profesor de filosofía y yo [...] hacemos buen equipo [...]. Cada sesión trabajábamos el tema que él había elegido y como los chicos respondieron muy bien, le sugerí que en este semestre les dejáramos a ellos la elección de temas, él aceptó inmediatamente" (P10).

El que los profesores puedan trabajar de manera conjunta el programa de atención tutorial ejemplifica la flexibilidad del modelo educativo y, con ello, la libertad para definir su organización y dinámica. La relación con el otro se hace rompiendo esquemas jerárquicos y abriendo espacios para que los estudiantes compartan la autoridad en la definición de las temáticas, así como para el reconocimiento y gestión de sus saberes, necesidades, realidades e inquietudes. El fundamento de esta relación lo consideran como sigue:

Busco incidir en la importancia de la reflexión, de la toma de decisiones y asumir con entereza los riesgos [...] La reflexión filosófica [...] no es la mera apropiación del conocimiento, sino conocernos a nosotros y en función de éste tomar decisiones (P4).

... yo hablo de que seas consciente del daño que te estás haciendo o del beneficio que te estás llevando a tu vida personal [...] y tiene que ver con una ética personal [...] mi verdadera personalidad la formo yo solo con todo lo que tengo a mi alrededor, hasta de mis cuates (P10).

En ambos casos se busca que los jóvenes reflexionen sobre sí mismos a partir de la interpelación y el cuestionamiento que les produce lo escuchado, comprendido, aprendido de otros al respecto de esquemas y códigos de actuación que han orientado su vida, de tal modo que con libertad logren transformarlas en beneficio propio.

Con estos fundamentos, prácticas y códigos orientan el pensar y actuar de los jóvenes para reconocerse e ir constituyéndose como sujetos:

Me limito a escuchar y orientar en las posibilidades que se tienen, respetando absolutamente la decisión del estudiante. [...] trato de reconocerme, respetarme, aceptarme como soy. Esta misma relación la aplico con el otro; al no emitir juicios el otro se siente con la confianza de hablar, de mostrarse hasta dónde considere pertinente; como retroalimentación está el espacio de escucha [...] el joven no está buscando que le digan qué hacer, [...] al hablar descubre por sí mismo lo que quiere (P4). 
Ellos eligen, nosotros acompañamos, escuchamos y respetamos [...], no permitimos faltas de respeto, mostramos que hay que aprender a convivir [...], ellos se dan cuenta que somos francos y honestos y nos dicen: "Haber usted diga ¿qué está sintiendo? o ¿usted habla con sus hijos igual?". Igualito, porque es un modo de vida (P10).

En un principio todos hablábamos, nadie escuchaba, después empezamos a levantar la mano porque ya teníamos más confianza, ahora ya habla uno y todos lo escuchamos [...] porque sabemos que las demás personas te nutren para complementar tu comentario [...] es un espacio abierto donde se trabajan cosas muy personales, espirituales, o sea conocernos a nosotros mismos (J32).

La maestra me ayudó mucho con libros y experiencias que ella nos comparte y nosotros compartimos. Me ayudó a conocerme a mí mismo [...] reconozco mis capacidades y dejo de sentirme frustrado, triste, enojado o deprimido (J33).

El Filos-café se organiza por doble vía: una vertical, al proceder de los fundamentos, acuerdos iniciales, estructura y dirección definidos por los profesores, y otra horizontal, en la que ambos actores se relacionan en un plano ontológico de igualdad y respeto a las diferencias.

Los profesores, al ser objeto de su propio cuidado y establecer reglas de acción ética para consigo mismos, dan cuenta de su propio "modo de vida" y se vuelven dignos de ser ejemplo para los demás. La coherencia vital de los profesores habilita a los jóvenes para voltearse a ver como seres importantes, con la capacidad de asumir sus reflexiones, decisiones o cambios. Entre las prácticas de cuidado de sí mismo que el Filos-café hace posibles, podemos mencionar:

-Prácticas de escucha. Vividas como un proceso que pasa del caos y desconfianza al silencio atento, seguridad y estructura; también producen un mayor dominio en los afectos, discreción ética y confianza hacia los otros.

-Prácticas del habla. Temáticas que interesan, preocupan, conmueven e identifican a los jóvenes; propician una incitación para hablar, pero esta se orienta y toma fuerza por la forma en que los profesores se muestran ante ellos. Sin embargo, solo después de que estos son confrontados en la coherencia de su pensar, decir y hacer, lo compartido toma sentido y logra ser considerado en los cambios personales; se construye, así, un pacto a partir de las vidas compartidas (Foucault, 2002, p. 387). Con esta condición, los jóvenes aprenden a hablar y dan lugar a la verdad que otro emite, a la dirección que se establece, a la verdad sobre sí mismo y a la autonomía respecto a las transformaciones que se deciden hacer.

-Prácticas de lectura. Con ellas se producen ejercicios del pensamiento y de desplazamiento hacia la experiencia de los sujetos; por ello, son objeto de conocimiento y transformación, sobre todo cuando se traslada a prácticas del habla.

Algunos efectos producidos a partir de estas prácticas se aprecian en uno de los estudiantes:

... ahora puedo ver qué virtudes y defectos tengo para aprovecharlos; saber esto me ayuda en mi autoestima. Decido entrar a todas mis clases [...], intento fijar bien mis metas como terminar la prepa, ser independiente desde ahorita, por eso trabajo de cocinero [...], quiero acabar una carrera en gastronomía, pero también quiero ser músico porque de esa forma puedo ser más libre (J33). 
Mediante las prácticas realizadas en el Filos-café, este joven se mira, logra nombrar sus sentimientos e identifica un aporte vital. Conocerse le brinda unidad, tanto para configurar un mayor dominio de su voluntad frente a otros como para definir con mayor claridad las metas que pretende, incluyendo la reorganización de las acciones que le permitan concretarlas. Asimismo, establece consigo una relación más autónoma, que si bien atiende el ámbito económico-profesional, también lo hace en aspectos creativos y sensibles desde los cuales se quiere expresar y configurar la vida.

Las prácticas y transformaciones efectuadas por los jóvenes se soportan en tres tipos de códigos:

Racionales. Producen codificaciones verbales y reflexiones colectivas para comprender y confrontar diversas realidades; por ello, este espacio desafía sus modos de pensar y actuar al descubrir, imaginar y construir posibilidades de solución, acción o lucha.

Éticos. Construidos en la gestión conjunta de este espacio, en el compartir la autoridad; en el reconocimiento y respeto de sus diferencias y decisiones; en la libertad de cada sujeto para dar cuenta de sí y decidir sobre los cambios que pretende hacer.

Estéticos. Configurados desde las necesidades, intereses e inquietudes que emergen de los jóvenes; en las formas de abordaje; en la suspensión momentánea de lo que se dice ser; en la imaginación, deseo, creatividad, libertad para construir nuevos parámetros de actuación, incluida la movilización o afirmación de su identidad.

Con este microdispositivo pedagógico se aprecia una estructura que orienta elementos teóricos, prácticos y estratégicos para crear, articular y conducir múltiples relaciones. En él se activa un espacio para el encuentro intersubjetivo, en el cual los sujetos pueden dar cuenta de sí rompiendo con códigos, hábitos, rutinas, relaciones que los fragmentan o dispersan y dando lugar a nuevas formas de ser elegidas con libertad, en las cuales pueden asumirse como actores de su labor educativa, como gobernantes de sus ámbitos cotidianos, de sus necesidades corporales, de aquello que se quiere superar y que les permite prefigurar un devenir otro.

\section{Del semáforo a los festivales escolares}

Durante un tiempo indefinido distintas expresiones provenientes del espectáculo circense, en particular del malabarismo realizado en las calles, irrumpe en el espacio escolar de la preparatoria Otilio Montaño: "Los malabares se pusieron de moda en la escuela por unos chicos que los practicaban, muchos de ellos se paran en el semáforo del Periférico y San Fernando, ahí piden dinero porque no tienen recursos y sí son muy adictos" (P6).

En un crucero urbano del sur de la ciudad, jóvenes estudiantes, o no, realizan prácticas que implican concentración mental, agilidad física, dominio psicomotriz; se producen ritmos, rutinas y hábitos mediante los cuales los jóvenes se comunican, reconocen y buscan transmitir algo al espectador. En este fragmento de la calle comparten saberes, valores y formas de relación, de sentir y ver el mundo.

Portadores de esos aprendizajes, algunos de aquellos jóvenes también van a la escuela, pero su estar en ella se vive desde lógicas y ritmos distintos, y producen ciertas desestabilizaciones en el ordenamiento escolar, reto creativo para muchos profesores o cierre perceptivo y de acción para otros. En seguida se presentan las acciones que una profesora de historia logra emprender junto con estos jóvenes: 
Unos chicos no entraban a clase porque se la pasaban haciendo malabares en el patio [...] tomando la fecha del 2 de octubre, hicimos un homenaje con un batallón circense a los estudiantes que mataron para darle un sentido histórico. Investigaron y abrieron un taller de malabar para los que no sabían, aunque duró poco por sus dispersiones. [...] Teníamos reuniones con un equipo base y luego ellos lideraban a otros chicos. El otro evento fue el día del estudiante, les dije: "Ya están muy feas las canchas, hay que pintarlas" [...] vinculado con Topilejo (P6).

Como acto urgente, la profesora cambia su registro perceptivo para establecer otras líneas de relación con su disciplina y de comunicación con los estudiantes, y reconoce aquellos marcadores que definen su visión del mundo, ritmos vitales e intereses. Por ello, abre espacios para la escucha y para mantener conversaciones y trabajo en común.

Ubicada entre lo escolar y lo no escolar, la profesora tiene actitudes de complicidad y creatividad para construir distintos vínculos; en uno busca direccionar el proceso de conocimiento a partir de la indagación reflexionada de los acontecimientos, así como de la identificación entre pares como sujetos históricos capaces de movilizar sus realidades. Un estudiante de veinte años de edad, participante de aquellas actividades, comparte:

Solo era llamar la atención, por eso hicimos el taller de malabar [...]. El 2 de octubre se dio la presentación, pláticas sobre el tema, hicimos una rutina, una marcha, pusimos música y terminamos. El día del estudiante nos presentaron como grupo organizador, se hicieron malabares y luego se pintó la barda mientras entraron las bandas de rackabilly, hip hop, first time y de ska (J4).

Aunque los jóvenes logran desplazarse por algunos aspectos históricos, políticos, culturales y comunitarios, el foco del microdispositivo se encuentra en la posibilidad de mostrarse frente a los otros a partir de la creación y yuxtaposición efímera dentro del espacio escolar, de un espacio heterotópico (Foucault, 2008), donde aquella esquina de la ciudad se hace presente en el territorio escolar con rasgos lúdicos, festivos y estéticos.

En ese lugar, el tiempo escolar se suspende y el ritmo lo marcan los cuerpos, los objetos en movimiento, las expresiones musicales, los códigos y colores plasmados en la pared; se produce una horizontalidad en las relaciones que genera una construcción ética en la que cabe la camaradería y el bien común.

Asimismo es importante comunicar los saberes que le dan sentido y los empodera: los procedentes de lenguajes, ritmos, cosmovisiones, resistencias de distintas expresiones culturales, y los que manifiestan otras formas de sensibilización ante el juego, el placer y el dominio corporal, como mediadores de adopción estética para el existir.

De aquel lugar algunos estudiantes toman lo que los enriquece, producen cambios subjetivos y toman decisiones al respecto de sí:

... Me dio más seguridad, obtuve facilidad para hablar y perder la timidez [...] quiero hacer cosas, conocer lugares [...] Si quiero trabajar y dejar de estudiar siento que tengo más posibilidades (J4).

De ese grupo de chicos una está en la universidad. [...] Había un chico que tenía problemas incluso para hablar, superadicto, ex convicto y terminó la prepa, hizo muy buen problema eje (P6).

Aunque estos microdispositivos tienen una temporalidad efímera, contribuyen a movilizar en algunos de los jóvenes decisiones que los conducen a otros espacios 
y formas de relación, como el terminar la escuela, romper con tramas autodestructivas en las que son subestimados por sí mismos o por los demás respecto a sus capacidades y posibilidades, y en las que hay otras búsquedas y ampliación de experiencias vitales.

Como estos microdispositivos se construyen desde la urgencia, tanto la reflexión como la acción teórico-práctica es menos profunda. Aun cuando se pretende producir un vínculo disciplinar dotado de sentido, destaca la presencia de lo no escolar; por lo tanto, las prácticas y los saberes puestos en juego buscan visibilizar ese otro mundo en el que un grupo de estudiantes está inmerso. Y no obstante la fugacidad de la conexión, son posibles ciertos cambios con los que se acercan a la amplificación de sus oportunidades y al reconocimiento de sus capacidades y habilidades para decidir hacer algo más constructivo con su vida.

\section{CONCLUSIONES O VISIBILIDADES PARA LA TRANSFORMACIÓN}

Después de lo abordado, nuestro supuesto se afirma, al destacar el papel fundamental de los profesores, quienes centran su atención en las condiciones de los estudiantes y en las posibilidades de acción que permite el modelo educativo. En este sentido, los microdispositivos presentados dejan ver elementos importantes a ser considerados en un quehacer pedagógico transformador.

\section{Visibilidades didácticas}

Los microdispositivos con mayor andamiaje se producen por el trabajo teórico-práctico de los profesores sobre su disciplina, expresado en la sistematización programática, en el uso creativo del tiempo y en el aprovechamiento de los entornos educativos y de técnicas participativas que transforman las actividades en estructuras móviles, flexibles, ricas en aportaciones para atender las particularidades de los grupos y los sujetos.

Un punto central que anima la configuración de estos microdispositivos es la presencia dentro del territorio escolar de otros mundos, de rasgos y desafíos frente a las épocas portados por la alteridad de los estudiantes, quienes logran movilizar la mirada, saberes, posturas de los profesores frente a la realidad. Desde este movimiento, los objetos teóricos se ven amplificados y resignificados; los procesos de enseñanza toman un sentido más real para los jóvenes por su continua recurrencia, lo que no implica descartar lo ya sabido y actuado del dispositivo escolar, pues juega un papel importante en la reflexión y acción para prefigurar caminos emergentes de formación.

Una lectura de lo que sucede en el espacio escolar y sus habitantes permite a los profesores aprovechar libremente los elementos arquitectónicos, materiales y recursos disponibles para crear espacios y prácticas pedagógicas más o menos estructuradas y responder a eso que no tiene tanta explicación, a ritmos, lógicas y sentidos que buscan conectarse con lo ya conocido.

En estos procesos se identifica un cariz de dinamismo, una sensibilidad distinta ante lo que se puede hacer con las emociones, el cuerpo, el intercambio participativo, la imaginación, la libertad, el silencio y la creación de códigos comunicativos comunes. Es la relación ética con el otro la que soporta esta sensibilidad o reactivación estética. 
La transmisión, creación y apropiación de conocimientos se transforma para los sujetos pedagógicos en saberes que les brindan nuevos sentidos, pues al convocar dimensiones notablemente humanas, se desata la identificación, el deseo de pensar $\mathrm{y}$ poner en acto aquello que se ha aprehendido y volverlo ethos.

\section{Visibilidades de autoridad pedagógica}

Los microdispositivos pedagógicos que posibilitan prácticas para el cuidado de sí mismo dejan ver un estatuto de igualdad humana compartido mediante el reconocimiento de problemas, sensibilidades, necesidades, fragilidades, ignorancias, etcétera. Se abren conexiones de comunicación, confianza y aprendizaje intergeneracional, en las que la dirección y jerarquía de los profesores no se pierde. La disciplina escolar es experimentada sin violencia o anulación de los sujetos, en la disposición de trabajos y acuerdos colectivos, en la orientación sin autoritarismo, en el respeto y la estructuración de la libertad habilitada para crecer.

\section{Visibilidades de fractura a la normalización}

El interés de los profesores por sus estudiantes hace del dispositivo escolar un lugar de fractura de los procesos de normalización, ya que, al atender relaciones, procesos, límites entre sujetos con diferentes valores, saberes, intereses, no se homogeneiza ni se excluye, sino que se recrea el lugar de las diferencias y, así, el lugar del devenir.

Los procesos vividos dan, en mayor o menor medida, la oportunidad de decir libremente quien se es, lo que se piensa, las apuestas efectuadas y las verdades elegidas, al exponer los alcances, límites y retos en la apropiación y el dominio ético, así como los niveles de ruptura frente a estereotipos, designios históricos o culturales configurados sobre ellos y las nuevas pautas estéticas que se pretenden.

Por último, consideramos que, aun cuando en la escuela prevalecen inercias, indiferencias, resistencias al cambio, que dificultan su capacidad de respuesta ante las situaciones del presente, también es posible descolocarla y hacer que vaya más allá de las lógicas estatales e institucionales.

En la osadía de los profesores vemos la capacidad de construir ricas experiencias formativas y de transmisión cultural en las cuales los estudiantes, además de hallar espacios confiables, abiertos e incluyentes, logren reconfigurar sus narrativas biográficas y, con ello, una posición distinta para actuar y estar en el mundo. Desde esta perspectiva, pasar por la escuela puede ser más que un momento en sus vidas y volverse un valioso acontecimiento que marque la diferencia en la configuración de sus proyectos de existencia.

\section{REFERENCIAS BIBLIOGRÁFICAS}

Álvarez Marín, Ana María (2012). Dispositivos de formación para la participación ciudadana de la licenciatura en Pedagogía de la FES Aragón. Tesis de maestría. UNAM, FES Aragón, DEPI, México. Recuperado de http://tesis. unam.mx/F/?func=findb\&find_code=WTT\&request=Dispositivos+de+forma ci\%C3\%B3n+para+la+participaci\%C3\%B3n+ciudadana\&local_base=TES01

Castañeda Salgado, Adelina (2004). Dispositivos de formación y uso de tecnologías. Espacios de articulación de saber, poder y subjetivación. En Adelina Castañeda, Cecilia Nava y Teresa Yurén. Formación, distancias y subjetividades: 
nuevos retos de la formación en la globalización (pp. 15-35). México: Noriega Editores/Universidad Autónoma de Morelos.

De Alba Ceballos, Alicia (2011). La educación en el siglo XXI. La importancia de abrir los espacios de la normalidad. Una reflexión a partir de la obra de Michel Foucault. En Alicia de Alba Alicia y Manuel Martínez (coords.). Pensar con Foucault. Nuevos horizontes e imaginarios en educación (pp. 85-116). México: UNAM-UAZ-IISUE, col. Educación. Debate e imaginario social, serie Teoría y educación.

Deleuze, Gilles (1999). ¿Qué es un dispositivo? En Barbier et al. Michel Foucault, filósofo. Barcelona: Gedisa.

Deleuze, Gilles (1987). Foucault. México: Paidós Studio.

Deleuze, Gilles y Guattari, Félix (2002). Mil mesetas. Capitalismo y esquizofrenia. Valencia: Pre-textos.

Duschatzky, Silvia (2007). Maestros errantes. Experimentaciones sociales en la intemperie. Buenos aires: Paidós.

Duschatzky, Silvia y Sztulwark, Diego (2011). Imágenes de lo no escolar. En la escuela y más allá. Buenos Aires: Paidós.

Elizondo Huerta, Aurora (2004). La construcción de un espacio intercultural mediante dispositivos de investigación-implicación. En Adelina Castañeda, Cecilia Nava y Teresa Yurén. Formación, distancias y subjetividades: nuevos retos de la formación en la globalización (pp. 37-53). México: Noriega Editores/ Universidad Autónoma de Morelos.

Estrada, Marcos y Araújo-Olivera, Stella (2008). Juventud y proyecto de vida. El caso de un dispositivo de nivel medio superior. En Teresa Yurén y Citlali Romero (coords.). La formación de los jóvenes en México. Dentro y fuera de los límites de la escuela (pp. 99-124). México: Casa Juanpablos.

Foucault, Michel (2013). La inquietud por la verdad. Escritos sobre la sexualidad y el sujeto. Buenos Aires: Siglo XXI, serie Fragmentos Foucaltianos, 2.

Foucault, Michel (2010). El coraje de la verdad: el gobierno de sí y los otros II. Curso en el Collége de France (1983-1984). Buenos Aires: Fondo de Cultura Económica.

Foucault, Michel (2008). Topologías. (Dos conferencias radiofónicas.) Utopías y heterotopías. El cuerpo utópico. Fractal, vol. 48, núm. 13 (12), pp. 39-62. Recuperado de http://www.mxfractal.org/RevistaFractal48MichelFoucault.html

Foucault, Michel (2002). Hermenéutica del sujeto. Curso del Collége de France (1981-1982) (2 ${ }^{\mathrm{a}}$ ed.). México: Fondo de Cultura Económica.

Foucault, Michel (2001). Fearles Speech. EUA: Semiotext.

Foucault, Michel (1999). Ética, estética y hermenéutica, vol. III. Barcelona: Paidós Básica.

Foucault, Michel (1992). Nietzsche, la genealogía, la historia. En Microfísica del poder (3a ed.). Madrid: Ediciones La Piqueta.

Foucault, Michel (1990). Tecnologías del Yo y otros textos afines. Barcelona: Paidós/ICE/UAB.

Foucault, Michel (1967/1969). Nietzsche, Freud, Marx. Ttítulo original: Nietzsche. Cahiers de Royaumont. Philosophie núm. VI, París. En dossier Nietzsche, 125 años, Eco, vol. 113, núm. 5, t. 19, n. 5-6-7. Bogotá. Recuperado de http:// www.academia.edu/9274924/Foucault_Michel_Nietzsche_Freud_Marx 
Grüner, Eduardo (1967/1969). Prólogo: Foucault: una política de la interpretación. En Foucault, Nietzsche, Freud, Marx. Título original: Nietzsche. Cahiers de Royaumont. Philosophie 6, París. En dossier Nietzsche, 125 años, Eco, vol. 113, núm. 5, t. 19, n. 5-6-7, Bogotá. Recuperado de http://www.academia. edu/9274924/Foucault_Michel_Nietzsche_Freud_Marx

Guerrero Salinas, María Elsa (2000). La escuela como espacio de vida juvenil. Dimensiones de un espacio de formación, participación y expresión de los jóvenes. Revista Mexicana de Investigación Educativa, vol. 5, núm. 10.

IEMSDF (2006). Proyecto Educativo. Sistema de Bachillerato del GDF. México: GDF/SDS/IEMS.

Ramírez Montes de Oca, Mónica C. (2012). Ser joven en el bachillerato. Una experiencia configurada desde la Escuela Preparatoria Oficial del Estado de México No. 115. Tesis de maestría. UNAM, FFyL, México.

Souto, Marta et al. (1999). Grupos y dispositivos de formación. Buenos Aires: Universidad de Buenos Aires, FFyL, col. Novedades educativas.

Tapia García, Guillermo (2012). Perspectivas de los "nuevos estudiantes" al ingresar al bachillerato en el Bajío mexicano. En Eduardo Weiss et al. (coord.). Jóvenes y bachillerato (pp. 63-96). México: ANUIES, col. Biblioteca de la Educación Superior, serie Investigaciones.

Valencia Reyes, Alicia (2008). El ethos juvenil en los bordes del dispositivo escolar. El caso de un dispositivo de educación media superior. En Teresa Yurén y Citlali Romero (coords.). La formación de los jóvenes en México. Dentro y fuera de los límites de la escuela (pp. 125-141). México: Casa Juanpablos.

Weiss, Eduardo et al. (coord.) (2012). Jóvenes y bachillerato. México: ANUIES, col. Biblioteca de la Educación Superior serie investigaciones.

Weiss, Eduardo et al. (2009). Jóvenes y bachillerato en México: el proceso de subjetivación, el encuentro con los otros y la reflexividad. Revista Propuesta Educativa, vol. 18, núm. 32, pp. 83-94. Recuperado de http://www.propuestaeducativa.flacso.org.ar/archivos/articulos/18.pdf

Yurén, Teresa (2008). Dispositivos de formación sociomoral, mecanismos de exclusión y fugas autoformativas. En Teresa Yurén y Citlali Romero (coords.). La formación de los jóvenes en México. Dentro y fuera de los límites de la escuela (pp. 25-47). México: Casa Juanpablos. 